\title{
A Singularity Free Solution of the Maxwell-Einstein Equations
}

\author{
P. Dolan
}

Department of Mathematics, Imperial College, London

\author{
Received March 1, 1968
}

\begin{abstract}
It is found that the massless charged particle permanently at rest at the origin of spherical polar coordinates in Lovelock's interpretation [1] of Robinson's solution of the Einstein-Maxwell equations [2] will repel all charged test particles, irrespective of the sign of their charges. By a global embedding of the space-time in a flat 6 -space we find an absence of singularities where point-charges or point-masses might be located. With the use of the Newman-Penrose method of spin-coefficients [6] it is shown that all the Robinson solutions [2] represent constant electromagnetic fields.
\end{abstract}

\section{Introduction}

The Einstein-Maxwell equations in the absence of sources are

$$
\left.\begin{array}{l}
R_{k}^{i}-\frac{1}{2} R \delta_{k}^{i}+F^{i j} F_{j k}+* F^{i j} * F_{j k}=0 \\
F_{; j}^{i j}=0, \quad * F_{; j}^{i j}=0
\end{array}\right\}
$$

where $R_{k}^{i}$ is the Ricci tensor, $F_{i j}=-F_{j i}$ the electromagnetic field tensor and $* F_{i j}=1 / 2(-g)^{1 / 2} \varepsilon_{i j k l} F^{k l}$ its dual. Covariant differentation with respect to the metric tensor $g_{i j}$ is denoted by a semi-colon. RoBINson [2] has found the following solution of (1.1):

$$
\begin{aligned}
& d s^{2}=\left(\frac{e^{2}}{r^{2}}\right)\left(c^{2} d t^{2}-d r^{2}-r^{2} d \theta^{2}-r^{2} \sin ^{2} \theta d \phi^{2}\right) \\
& F_{i j}=e\left(\xi_{i j} \cos \beta+* \xi_{i j} \sin \beta\right), \\
& \xi_{i j}=\left(\frac{1}{r^{2}}\right) \delta_{i j}^{10}
\end{aligned}
$$

where $e$ and $\beta$ are disposable constants.

The solution (1.2), if written in terms of the new coordinates

$$
\tilde{x}^{0}=e(c t-r), \tilde{x}^{1}=\frac{e}{r}, \quad \tilde{x}^{2}=e(\pi / 2-\theta), \quad \tilde{x}^{3}=e \phi,
$$

becomes

$$
\left.\begin{array}{l}
d s^{2}=e^{-2}\left(\tilde{x}^{1} d \tilde{x}^{0}\right)^{2}+2 d \tilde{x}^{0} d \tilde{x}^{1}-\left(d \tilde{x}^{2}\right)^{2}-\left(\cos \left(\tilde{x}^{2} / e\right) d \tilde{x}^{3}\right)^{2} \\
F_{i j}=\frac{1}{e} \delta_{i j}^{01} \cos \beta+\frac{1}{e} \delta_{i j}^{23} \cos \left(\tilde{x}^{2} / e\right) \sin \beta
\end{array}\right\}
$$

in which the Maxwell field is now expressed independently of the coordinate $r$ [2]. A further coordinate transformation

$$
2 c T=\tilde{x}^{0}+\tilde{x}^{1}, \quad 2 X=\tilde{x}^{0}-\tilde{x}^{1}, \quad Y=\tilde{x}^{2}, \quad Z=\tilde{x}^{3}
$$

11 Commun. math. Phys., Vol. 9 
changes (1.3) to

$$
\begin{gathered}
d s^{2}=e^{-2}(c T-X)^{2} d(c T+X)^{2}+c^{2} d T^{2}-d X^{2} \\
-d Y^{2}-\cos ^{2}(Y \mid e) d Z^{2} \ldots
\end{gathered}
$$

If we write $E_{a}=F_{0 a}$ and $H_{a}=* F_{0 a}(a=1,2,3)$ for the electric and magnetic fields then to the first order in $\frac{1}{e}$ the solution (1.4) becomes

and

$$
d s^{2}=c^{2} d T^{2}-d X^{2}-d Y^{2}-d Z^{2}
$$

$$
\underline{E}=\left(\frac{1}{e} \cos \beta, 0,0\right), \quad \underline{H}=\left(\frac{1}{e} \sin \beta, 0,0\right) .
$$

Since the electromagnetic field in this approximation represents a constant electric field or a constant magnetic field or a superposition of both [2] it is rather surprising to find in [1] an exact interpretation of a special case of (1.2) as a point-charge singularity and its field.

LovELOCK [1] has considered the case $\beta=0$ and has interpreted it as the field of "a massless particle of charge $e$ at rest at the origin for all time". He is assuming, of course, that the coordinate $r$ has its usual significance, when $t=$ constant, of giving a one-parameter family of spheres $r=$ constant centred on $r=0$, a degenerate sphere or mere point, and that $\theta$ and $\phi$ coordinatize all the non-degenerate spheres in the usual way. Unfortunately, in the space-time (1.2) all such non-degenerate space-like spheres have metric

$$
d s^{2}=-e^{2}\left(d \theta^{2}+\sin ^{2} \theta d \phi^{2}\right)
$$

and so each coincides with a spherical surface of constant radius $e$. It will be shown that the space-time is globally the Cartesian product of a space-like sphere $S^{2}$ and the surface of a one sheeted hyperboloid of indefinite metric. The centre of each $S^{2}$ will not lie on the space-time and so cannot be $r=0$ even if this 3 -space had degenerated into a timelike curve. In fact, to locate the centre of $S^{2}$ the space-time must be embedded in a higher dimensional flat space (of minimal dimension 6). Thus the assumption that $r=0$ is a degenerate 3 -space and so represents the history of a particle in a spherically symmetrical situation has no mathematical basis.

It will also be shown by a physical argument that this hypothesis about the usual kind of spherical symmetry cannot be sustained.

Putting

\section{Motion of a Charged Test Particle}

$$
x^{0}=c t, \quad x^{1}=r, \quad x^{2}=\theta, \quad x^{3}=\phi
$$

it follows easily that the only non-vanishing Christoffel symbols for the metric (1.2) are

$$
\begin{aligned}
& \left\{\begin{array}{cc}
0 & 1 \\
0 & 1
\end{array}\right\}=\left\{\begin{array}{cc}
1 & 0 \\
0 & 0
\end{array}\right\}=\left\{\begin{array}{cc}
1 & 1 \\
1 & 1
\end{array}\right\}=-1 / r \\
& \left\{\begin{array}{cc}
2 & 3
\end{array}\right\}=-\sin \theta \cos \theta,\left\{\begin{array}{cc}
3 \\
2 & 3
\end{array}\right\}=\cot \theta .
\end{aligned}
$$


A test particle of mass $m$ and charge $Q$ experiences a mechanical force $Q F^{i j} U_{j}$, where $U^{i}=\dot{x}^{i} \equiv d x^{i} / d s$ is its velocity 4 -vector. By (1.2), with $\beta=0$,

$$
\begin{aligned}
F_{j k} U^{k} & =e \xi_{j k} U^{k} \\
& =\frac{e}{r^{2}} \delta_{j k}^{10} U^{k} \\
& =\left(-\frac{e}{r^{2}} U^{1}, \frac{e}{r^{2}} U^{0}, 0,0\right)
\end{aligned}
$$

and so $Q F^{i j} U_{j}=Q g^{i j} F_{j k} U^{k}$

$$
=\left(-\frac{Q}{e} U^{1},-\frac{Q}{e} U^{0}, 0,0\right) .
$$

Writing $\mu=Q / m e$ the equations of motion of the test particle are

$$
\begin{aligned}
& \ddot{t}-\frac{2}{r} \dot{r} \dot{t}=-\mu \dot{r}, \\
& \ddot{r}-\frac{1}{r}\left(\dot{r}^{2}+c^{2} \dot{t}^{2}\right)=-\mu c \dot{t}, \\
& \ddot{\theta}-\sin \theta \cos \theta \dot{\phi}^{2}=0, \\
& \ddot{\phi}-2 \cot \theta \dot{\theta} \dot{\phi}=0 .
\end{aligned}
$$

The conditions $\dot{\theta}=0, \theta=\pi / 2$ can be maintained permanently if $\ddot{\phi}=0$. There is no loss of generality in taking these initial conditions. We can also take

$$
\phi=h s, \quad h \text { constant . }
$$

The first of equations (2.2) can be integrated to give

$$
\dot{t}-\mu r=K r^{2},
$$

where $K$ is an arbitrary constant. From the metric (1.2) we get

$$
\dot{r}^{2} / r^{2}=c^{2}(K r+\mu)^{2}-\alpha^{2}
$$

on putting $\alpha^{2}=h^{2}+1 / a^{2}$.

Equation (2.5) can be rewritten as

where $\varrho=r+\mu / K, b=\alpha / K c$.

$$
d \varrho / d t=\frac{c \sqrt{\varrho^{2}-b^{2}}}{\varrho},
$$

The initial conditions are $t=0, r=r_{0}$ (or $\left.\varrho=\varrho_{0}=r_{0}+\mu / K\right), d r / d t=V$. Hence

$$
c t+V \varrho_{0} / c=\left(\varrho^{2}-\varrho_{0}^{2}\left(1-V^{2} / c^{2}\right)\right)^{1 / 2}
$$

which gives valid equations of motion if and only if

$$
\varrho^{2} \geqq \varrho_{0}^{2}\left(1-V^{2} / c^{2}\right) \text {. }
$$

For a particle released from rest on the 2 -space $r=r_{0}$ at $t=0$ the last condition becomes

$$
r \geqq r_{0} .
$$


Thus, on Lovelock's ad hoc topological assumption about the family of surfaces $r=$ constant, all charged test particles are repelled from the apparent centre of force at $r=0$ irrespective of the sign of their charges ${ }^{1}$.

The case $\mu=0$ is considered by Lovelock in another paper [3].

In all cases the hypothesis about the usual kind of spherical symmetry does not lead to physically meaningful results.

\section{The Curvature Tensor and its Invariants}

From the metric (1.2) one can easily show that the only non-vanishing components of the Riemann tensor are

$$
R_{0110}=e^{2} / r^{4}, \quad R_{2323}=-e^{2} \sin ^{2} \theta .
$$

From which it easily follows that

Since

$$
R_{i j k l}+F_{i j} F_{k l}+* F_{i j} * F_{k l}=0 \text {. }
$$

$$
F_{i j ; k}=0, \quad * F_{i j ; k}=0
$$

can easily be verified for the Maxwell-Einstein field (1.2) we deduce from (3.2) that

$$
R_{i j k l ; m}=0 \text {, }
$$

i.e., the Riemann tensor is covariantly constant. The same applies also to any of its invariants formed by contraction and transvection with itself, the metric tensor and the tensor density $\varepsilon_{i j k l}$. Thus it is very likely that the space-time has no singular points. In $\S 4$ we shall confirm that this is so by a global embedding of (1.2) in a flat six-space.

\section{A Global Embedding of the Space-Time}

Theorem. The portion of space-time with (1.2) as metric can be imbedded locally in the pseudo-Euclidean 6-space $E_{6}$ of metric

$$
d s^{2}=d u^{2}+d v^{2}-d w^{2}-d x^{2}-d y^{2}-d z^{2}
$$

as part of the 4 dimensional submanifold given by

$$
\begin{aligned}
& u^{2}+v^{2}-v^{2}=1, \\
& x^{2}+y^{2}+z^{2}=a^{2} .
\end{aligned}
$$

Proof. Consider the coordinate transformation

$$
\begin{gathered}
u=c t / r, \quad v=\frac{c^{2} t^{2}-r^{2}-\frac{1}{4}}{r}, \quad w=\frac{c^{2} t^{2}-r^{2}+\frac{1}{4}}{r} \\
x=a \sin \theta \cos \phi, \quad y=a \sin \theta \sin \phi z=a \cos \theta
\end{gathered}
$$

which satisfies (4.2) and (4.3). (See (5)). If we write

$$
\begin{gathered}
z^{1}=u, z^{2}=v, z^{3}=w, z^{4}=x, z^{5}=y, z^{6}=z \\
\varepsilon_{1}=\varepsilon_{2}=1, \quad \varepsilon_{3}=\varepsilon_{4}=\varepsilon_{5}=\varepsilon_{6}=-1
\end{gathered}
$$

1 If the negative square root had been taken in (2.6) or if $t$ had been taken to be negative and decreasing or both the inequality (2.8) would still follow. 
and allow the capital letters $A, B, \ldots$ to have the range 1 to 6 we get

$$
\sum_{A=1}^{6} \varepsilon_{A} z^{A},{ }_{i} z^{A},{ }_{j}=g_{i j}
$$

where $g_{i j}$ is the metric tensor (1.2),

$$
x^{0}=c t, \quad x^{1}=r, \quad x^{2}=\theta, \quad x^{3}=\phi
$$

and a comma indicates partial differentiation with respect to the $x^{i}$.

Corollary. The submanifold of $M_{6}$ represented by (4.2) and (4.3) is inextendible and so represents a global embedding of the space-time metric (1.2).

Proof. As the sphere (4.3) is inextendible it is sufficient to consider only the one-sheeted hyperboloid of revolution (4.2). The families of straight lines (in $u, v, w$ space)

$$
\begin{array}{ll}
v+w=\lambda(1-u), & v-w=\frac{1}{\lambda}(1+u) \\
v-w=\mu(1-u), & v+w=\frac{1}{\mu}(1+u)
\end{array}
$$

lie completely on the surface (4.2) for all real values of $\lambda$ and $\mu$. (Of course, for $\lambda=0$ we interpret (4.7) to mean the straight line $v+w=0,1+u=0$ and for $\lambda= \pm \infty$ to mean the straight line $v-w=0,1-u=0$. Similar interpretations apply to $\mu=0$ and $\mu= \pm \infty)$. Each straight line is an inextendible manifold of one dimension and through every point of the hyperboloid (4.2) pass two generators, one from each family: In fact the two generators, through a point $(u, v, w)$ of $(4.2)$ determine it uniquely through the relations

$$
u=\frac{\lambda \mu-1}{\lambda \mu+1}, \quad v=\frac{\lambda+\mu}{\lambda \mu+1}, \quad w=\frac{\lambda-\mu}{\lambda \mu+1}
$$

because a line of the $\lambda$-system meets every $\mu$-generator once and only once and vice-versa [4]. Thus the set of all $\infty^{2}$ points on the hyperboloid is the same as the set of all points on the $\lambda$ - and $\mu$-systems of straight lines Hence (4.2) is inextendible.

From (4.4) we find that

$$
v-w=-\frac{1}{2 r}
$$

Treat a typical sphere $S^{2}$ (covered by $x, y, z$ coordinates) as a point in $u, v, w$ space then the locus $r=$ constant will represent the curve in which the plane (4.10) cuts the hyperboloid (4.2). For $r= \pm \infty$ we get the unique plane $v-w=0$; while for $r \rightarrow 0$ we get two distinct plane curves at either "end" of the hyperboloid. (To make this latter remark obvious the reader should note that the plane $(4.10)$ cuts the $v$-and $w$-axes in the points $\left(0,-\frac{1}{2 r}, 0\right)$ and $\left(0,0, \frac{1}{2 r}\right)$ respectively: Now if we let 
$r \rightarrow+0$ these points are $(0,-\infty, 0)$ and $(0,0,+\infty)$ respectively and if $r \rightarrow-0$ they are $(0,+\infty, 0)$ and $(0,0,-\infty)$. As we do not identify the ends of the $v$-and $w$-axes the two remote planes are distinct).

Thus the apparent repulsion of test particles away from $r=0$ in $\S 2$ was merely motion away from the infinite remote regions of the spacetime under the influence of the electromagnetic field.

\section{The Newman-Penrose Formalism}

Using the spin coefficient formalism of Newman and Penrose [6] it is very easy to show that the Robinson solution of the Einstein-Maxwell equations (1.1) represents a constant electromagnetic field.

The metric (1.2) can be written in terms of real Pfaffians $\omega^{\alpha}$, $\alpha=0,1,2,3$, as

$$
d s^{2}=\left(\omega^{0}\right)^{2}-\left(\omega^{1}\right)^{2}-\left(\omega^{2}\right)^{2}-\left(\omega^{3}\right)^{2} .
$$

A tetrad system of null vectors $\ell_{\mu}, n_{\mu}, m_{\mu}, \bar{m}_{\mu}$, of which $\ell_{\mu}, n_{\mu}$ are real and $m_{\mu}, \bar{m}_{\mu}$ are complex conjugate vectors, is defined by the relations

$$
\left.\begin{array}{rlrl}
\sqrt{2} \ell_{\mu} d x^{\mu} & =\omega^{0}+\omega^{3}, & \sqrt{2} n_{\mu} d x^{\mu}=\omega^{0}-\omega^{3} \\
\sqrt{2} m_{\mu} d x^{\mu}=\omega^{1}+i \omega^{2}, & \sqrt{2} \bar{m}_{\mu} d x^{\mu}=\omega^{1}-i \omega^{2}
\end{array}\right\}
$$

and the following orthonormality conditions are satisfied,

$$
\left.\begin{array}{l}
\ell_{\mu} \ell^{\mu}=m_{\mu} m^{\mu}=\bar{m}_{\mu} \bar{m}^{\mu}=n_{\mu} n^{\mu}=0, \\
\ell_{\mu} n^{\mu}=-m_{\mu} \bar{m}^{\mu}=1, \\
\ell_{\mu} m^{\mu}=\ell_{\mu} \bar{m}^{\mu}=n_{\mu} \bar{m}^{\mu}=n_{\mu} \bar{m}^{\mu}=0 .
\end{array}\right\}
$$

On introducing, as in [7] Debever's Pfaffians $\theta^{\alpha}, \alpha=0,1,2,3, Z^{\mathfrak{A}}$, $\mathfrak{A}=1,2,3$.

$$
\begin{aligned}
& \theta^{0}=\ell_{\mu} d x^{\mu}, \quad \theta^{3}=n_{\mu} d x^{\mu} \\
& \theta^{1}=m_{\mu} d x^{\mu}, \quad \theta^{2}=\bar{m}_{\mu} d x^{\mu} \\
& Z^{1}=\theta^{2} \wedge \theta^{3}, \quad Z^{2}=\theta^{0} \wedge \theta^{1}, \quad Z^{3}=\frac{1}{2}\left(\theta^{2} \wedge \theta^{3}-\theta^{1} \wedge \theta^{2}\right)
\end{aligned}
$$

the metric becomes $d s^{2}=2\left(\theta^{0} \theta^{3}-\theta^{1} \theta^{2}\right)$ and the Maxwell two-form $F=F_{\mu \nu} d x^{\mu} \wedge d x^{\nu}$ becomes

$$
F=F_{\mathfrak{A}} Z^{\mathfrak{A}}+\bar{F}_{\mathfrak{A}} \bar{Z}^{\mathfrak{A}}
$$

where " $\wedge$ " stands for Grassman multiplication on vectors in the cotangent space at any space-time point and

$$
F_{1}=F_{\mu \nu} \ell^{\mu} m^{\nu}, \quad F_{2}=F_{\mu \nu} \bar{m}^{\mu} n^{\nu}, \quad F_{3}=\frac{1}{2} F_{\mu \nu}\left(\ell^{\mu} n^{\nu}+\bar{m}^{\mu} m^{\nu}\right) .
$$

If we choose $\ell_{\mu}, n_{\mu}, m_{\mu}, \bar{m}_{\mu}$ to be the principal null vectors of the Maxwell field $F_{\mu \nu}$ then we must have

$$
F_{1}=F_{2}=0 \text {. }
$$


The spin coefficients $\alpha, \beta, \gamma, \varepsilon, \varkappa, \lambda, \mu, \nu, \pi, \varrho, \sigma, \tau$ are defined to be the complex scalars

$$
\begin{aligned}
& \varkappa=\ell_{\mu ; \nu} m^{\mu} \ell^{\nu}, \quad \pi=-n_{\mu ; \nu} \bar{m}^{\mu} \ell^{\nu}, \\
& \varepsilon=\frac{1}{2}\left(\ell_{\mu ; \nu} n^{\mu} \ell^{\nu}-m_{\mu ; \nu} \bar{m}^{\mu} \ell^{\nu}\right), \quad \varrho=\ell_{\mu ; \nu} m^{\mu} \bar{m}^{\nu}, \\
& \lambda=-n_{\mu ; \nu} \bar{m}^{\mu} m^{\nu}, \quad \alpha=\frac{1}{2}\left(\ell_{\mu ; \nu} n^{\mu} \bar{m}^{\nu}-m_{\mu ; \nu} \bar{m}^{\mu} \bar{m}^{\nu}\right) \\
& \sigma=\ell_{\mu ; \nu} m^{\mu} m^{\nu}, \quad \mu=-n_{\mu ; \nu} \bar{m}^{\mu} m^{\nu}, \\
& \beta=\frac{1}{2}\left(\ell_{\mu ; \nu} n^{\mu} m^{\nu}-m_{\mu ; \nu} \bar{m}^{\mu} m^{\nu}\right), \quad v=-n_{\mu ; \nu} \bar{m}^{\mu} n^{\nu} \\
& \gamma=\frac{1}{2}\left(\ell_{\mu ; \nu} n^{\mu} n^{\nu}-m_{\mu ; \nu} \bar{m}^{\mu} n^{\nu}\right), \quad \tau=\ell_{\mu ; \nu} m^{\mu} n^{\nu} .
\end{aligned}
$$

In a conformally flat space-time containing a Maxwell field the Bianchi identities become a set of first order partial differential equations involving the spin-coefficients and the quantities $F_{1}, F_{2}, F_{3}$ (equations A3 of the Newman-Penrose paper with vanishing conformal curvature tensor, i.e. $\left.\Psi_{0}=\Psi_{1}=\Psi_{2}=\Psi_{3}=\Psi_{4}=0\right)^{2}$. Since $\ell_{\mu}, n_{\mu}, m_{\mu}, \bar{m}_{\mu}$ are eigenvectors of $F_{\mu \nu}$ it is easy to deduce from the Newman-Penrose form of the Bianchi identities that $x=\lambda=\mu=\nu=\pi=\varrho=\sigma=\tau=0$.

Since $\ell_{\mu ; \nu} \ell^{\nu}=-\varkappa \bar{m}_{\mu}-\bar{x} m_{\mu}+(\varepsilon+\bar{\varepsilon}) \ell_{\mu}$ in general and $\varepsilon+\bar{\varepsilon}$ can be made zero by a change in scale on $\ell_{\mu}$ we deduce that $\ell_{\mu}$ is tangent vector to a geodesic when $x=0$. When $\nu=0$ the same is true of vector field $n_{\mu}$. From the vanishing of $\varkappa, \varrho, \sigma, \nu, \mu, \lambda$ we can also deduce that $\ell_{\mu}$ and $n_{\mu}$ are divergence-free, curl-free, shear-free and hypersurface-orthogonal in addition to being geodetic. Further, the equation $\ell_{\mu ; \nu} n^{\nu}=-\tau \bar{m}_{\mu}$ $-\bar{\tau} m_{\mu}+(\gamma+\bar{\gamma}) \ell_{\mu}$, in which $\gamma+\bar{\gamma}$ can be made zero by another change in seale of $\ell_{\mu}$, tells us that $\ell_{\mu}$ does not change as we move in the $n_{\mu}$ direction when $\tau=0$. A similar result for $n_{\mu}$ follows from $\pi=0$.

With spin-coefficients Maxwell's equations can be written as

$$
\left.\begin{array}{rl}
D F_{3}-\bar{\delta} F_{1} & =(\pi-2 \alpha) F_{1}+2 \varrho F_{3}-\varkappa F_{2} \\
D F_{2}-\bar{\delta} F_{3} & =-\lambda F_{1}+(\varrho-2 \varepsilon) F_{2}+2 \pi F_{3} \\
\delta F_{3}-\Delta F_{1} & =(\mu-2 \gamma) F_{1}-\sigma F_{2}+2 \tau F_{3} \\
\delta F_{2}-\Delta F_{3} & =-\nu F_{1}+(\tau-2 \beta) F_{2}+2 \mu F_{3}
\end{array}\right\}
$$

2 Omitting covariant derivatives of $F_{1}$ and $F_{2}$ they are

$$
\begin{aligned}
& \bar{F}_{3} F_{3} \nu-\bar{F}_{2} F_{3} \lambda-\bar{F}_{3} F_{2} \gamma+\bar{F}_{2} F_{2} \alpha=0, \\
& \bar{F}_{3} F_{3} \mu-\bar{F}_{2} F_{3} \pi-\bar{F}_{3} F_{2} \beta+\bar{F}_{2} F_{2} \varepsilon=0, \\
& \bar{F}_{3} F_{1} \gamma-\bar{F}_{2} F_{1} \alpha-\bar{F}_{3} F_{3} \tau+\bar{F}_{2} F_{3} \varrho=0, \\
& \bar{F}_{3} F_{1} \beta-\bar{F}_{2} F_{1} \varepsilon-\bar{F}_{3} F_{3} \sigma+\bar{F}_{2} F_{3} \varkappa=0, \\
& \bar{F}_{1} F_{3} \nu-\bar{F}_{3} F_{3} \lambda-\bar{F}_{1} F_{2} \gamma+\bar{F}_{3} F_{2} \alpha=0, \\
& \bar{F}_{1} F_{3} \mu-\bar{F}_{3} F_{3} \pi-\bar{F}_{1} F_{2} \beta+\bar{F}_{3} F_{2} \varepsilon=0, \\
& \bar{F}_{1} F_{1} \gamma-\bar{F}_{3} F_{1} \alpha-\bar{F}_{1} F_{3} \tau+\bar{F}_{3} F_{3} \varrho=0, \\
& \bar{F}_{1} F_{1} \beta-\bar{F}_{3} F_{1} \varepsilon-\bar{F}_{1} F_{3} \sigma+\bar{F}_{3} F_{3} \varkappa=0 .
\end{aligned}
$$


where

$$
\left.\begin{array}{rl}
D \psi & \equiv \psi_{; \mu} \ell^{\mu}, \quad \Delta \psi \equiv \psi_{; \mu} n^{\mu}, \\
\delta \psi & =\psi_{; \mu} m^{\mu}, \quad \bar{\delta} \psi=\psi_{; \mu} \bar{m}^{\mu}
\end{array}\right\}
$$

denote intrinsic derivatives. The vanishing of $\varkappa, \lambda, \mu, \nu, \pi, \varrho, \sigma, \tau, F_{1}, F_{2}$ gives $D F_{3}=\Delta F_{3}=\delta F_{3}=\bar{\delta} F_{3}=0$, i.e., $F_{3}$ is constant.

The reader is undoubtedly aware that the covariant constancy of $F_{\mu \nu}$ given by (3.3) (which makes all invariants formed from $F_{\mu \nu}$ by contraction and transvection with itself, the metric tensor and the tensor density $\varepsilon_{i j k l}$ constant) is not sufficient to characterize the field as unchanging in every physical aspect. To show this latter property, we needed the spin coefficient formalism.

\title{
6. Conclusion
}

There are no singularities in the space-time at which point charges or point masses might be located. The electromagnetic field on the spacetime in an exact treatment has been shown to be of the same nature (namely, a uniform field) as that reached approximately by RoBINson [2].

Acknowledgments. I wish to thank Dr. N. S. Swaminarayan for bringing Lovelock's work to my attention, Mr. M. Walker, and Professor F. A. E. Pirani for useful discussions. I am also very grateful to Professor A. H. TAUB for bringing the errors in the earlier version of the paper to our attention and for some very good advice. I am indebted to Mr. C. White for many helpful remarks on the NewmanPenrose formalism.

\section{Bibliography}

1. Lovelock, D.: A spherically symmetric solution of the Maxwell-Einstein equations. Commun. Math. Phys. 5, 257-261 (1967).

2. Robinson, I.: A solution of the Maxwell-Einstein equations. Bull. Acad. Pol. Sci. 7, 351-352 (1959).

3. Lovelock, D.: Weakened field equations in general relativity admitting an "unphysical" metric. Commun. Math. Phys. 5, 205-214 (1967).

4. McCrea, W. H.: Analytical geometry of three dimensions. Chap. VI, p. 43. London: Oliver and Boyd 1947.

5. Szekeres, P.: Embedding properties of general relativistic manifolds. Nuovo Cimento 43, 1062-1076 (1966).

6. Newman, E. T., and R. Penrose: An approach to gravitational radiation by a method of spin coefficients. J. Math. Phys. 3, 566-578 (1962).

7. Debever, R.: Le rayonnement gravitational. Cahier Phys. 18, 303-349 (1964).

\author{
P. Dolan \\ Imperial College of Science and Technology \\ University of London \\ Department of Mathematics \\ Exhibition Road \\ London-S.W. 7, Great Britain
}

